\title{
Distribución de metales pesados en sedimentos superficiales del Orinoco Medio, Venezuela
}

\author{
Aristide Márquez ${ }^{1,2, *}$, Orlys Garcia ${ }^{3}$, William Senior ${ }^{2,}$ Gregorio Martínez ${ }^{2}$ \\ y Ángel González ${ }^{4}$ \\ ${ }^{1}$ Departamento de Oceanografía, Instituto Oceanográfico de Venezuela, Universidad de \\ Oriente, Núcleo de Sucre, Cumaná, Venezuela. ${ }^{2}$ Programa de Doctorado en Oceanografía, \\ Universidad de Concepción, Chile. ${ }^{3}$ Programa de Postgrado en Química, IVIC, Caracas, \\ Venezuela. ${ }^{4}$ Instituto Limnológico, Universidad de Oriente, Caicara del Orinoco, Venezuela \\ Recibido: 09-05-11 Aceptado: 08-12-11
}

\section{Resumen}

La concentración total y las formas químicas de los metales pesados se determinaron en sedimentos superficiales del Orinoco Medio, Venezuela, utilizando una solución 25\% (v/v) de acido acético/solución $\mathrm{HNO}_{3}: \mathrm{HCl}: \mathrm{HClO}_{4}$ (3:2:1), espectrofotometría de absorción atómica con llama y técnica de vapor en frio. El rango de los valores totales variaron entre 8871 a $116.759 \mu \mathrm{gFeg}^{-1}$, 102,45 a 469,44 $\mu \mathrm{gMn} \mathrm{g}^{-1} ; 0,93$ a 17,64 $\mu \mathrm{gCu} \mathrm{g}^{-1} ; 4,46$ a $17,48 \mu g N i \mathrm{~g}^{-1} ; 2,46$ a 9,61 $\mu \mathrm{gCo} \mathrm{g}^{-1} ; 42,56$ a 181,45 $\mu \mathrm{gZn} \mathrm{g} \mathrm{g}^{-1} ; 1,29$ a 8,76 $\mu \mathrm{gCr} \mathrm{g}^{-1} ; 0,03$ a 0,74 $\mu \mathrm{gCd} \mathrm{g}^{-1}$ and 0,001 a 7,88 $\mu \mathrm{gPb} \mathrm{g}^{-1}$. Los metales están fuertemente asociados a la fracción que contiene los oxihidróxidos de hierro más resistente, sulfuros metálicos, minerales residuales refractarios y materia orgánica. Los rangos fueron: 7,50-99,29\% Fe; 7,75-66,34\% Mn; 22,55-98,89\% Zn; 22,85-91,36\% Ni; 4,20-85,03\% Cu; 16,76-85,48\% Co; 12,56-95,49 Cr; 7,50-99,29\% Pb; 2,03-85,48\% Cd. Los valores de metales adsorbidos en la superficie de las partículas, asociados con los carbonatos y los oxihidróxidos de manganeso reactivos variaron entre: 0,04-1,97\% Fe; 4,15-71,59\% Mn; 0,86-3,83\% Zn; 0-12,10\% Ni; 1,05-14,97\% Cu; 6,40-33,06\% Co; 1,03-5,08\% Cr; 0-1,78\% Pb; 0-22,97\% Cd. Las concentraciones totales de $\mathrm{Cu}, \mathrm{Ni}, \mathrm{Zn}$ y $\mathrm{Pb}$, son superiores a los reportados en la literatura para sedimentos no contaminados en algunas estaciones como los puertos de las ciudades de Cabruta y Caicara del Orinoco.

Palabras clave: Metales, sedimento, distribución, rio Orinoco.

\section{Heavy metals distribution on superficial sediments in Orinoco Medio, Venezuela}

\begin{abstract}
Total concentrations and chemical forms of metals in superficial sediments of the Middle Orinoco were determined with acetic acid solution $25 \%(\mathrm{v} / \mathrm{v}) / \mathrm{HNO}_{3}: \mathrm{HCl}: \mathrm{HClO}_{4}(3: 2: 1)$ solution, atomic absorption spectrometry with air acetylene flame and cold vapor technique. Total values ranged from 8871 to $116759 \mu \mathrm{gFeg}^{-1}, 102.45$ a $469.44 \mu \mathrm{gMn} \mathrm{g}{ }^{-1} ; 0.93$ to $17.64 \mu \mathrm{gCu} \mathrm{g}^{-1}$; 4.46 to $17.48 \mu \mathrm{gNi} \mathrm{g}^{-1} ; 2.46$ to $9.61 \mu \mathrm{gCo} \mathrm{g}^{-1} ; 42.56$ to $181.45 \mu \mathrm{gZn} \mathrm{g} \mathrm{g}^{-1} ; 1.29$ to $8.76 \mathrm{ggCr}^{-1} ; 0.03$ to $0.74 \mu \mathrm{gCd} \mathrm{g}^{-1}$ and 0.001 to $7.88 \mu \mathrm{gPb} \mathrm{g}^{-1}$. The metals were found to be strongly associated with

* Autor para la correspondencia: aristd@gmail.com
\end{abstract}


the residual fraction minerals, more resistant iron oxihidroxides, metallic sulfides, and organic matter. The values ranged from 7.50-99.29\% Fe; 7.75-66.34\% Mn; 22.55-98.89\% Zn; 22.8591.36\% Ni; 4.20-85.03\% Cu; 16.76-85.48\% Co; $12.56-95.49$ Cr; $7.50-99.29 \%$ Pb; $2.03-85.48 \%$ Cd). The values of metals adsorbed in the surface of particles, associated with carbonates and the reactive manganese oxihidróxidos varied from $0.04-1.97 \% \mathrm{Fe} ; 4.15-71.59 \% \mathrm{Mn}$; 0.86-3.83\% Zn; 0-12.10\% Ni; 1.05-14.97\% Cu; 6.40-33.06\% Co; 1.03-5.08\% Cr; 0-1.78\% Pb; 0-22.97\% Cd. The total concentration of $\mathrm{Cu}, \mathrm{Ni}, \mathrm{Zn}$ and $\mathrm{Pb}$ in some stations like the Cabruta and Caicara of Orinoco cities are superiors to the reported for non contaminated sediments.

Key words: Metals, sediment, distribution, Orinoco river.

\section{Introducción}

Una gran parte de los estudios realizados sobre suelos y sedimentos se refieren a la concentración total de metales, en especial cuando el estudio se encamina a concretar niveles de referencia de los metales o contenidos de fondo según diferentes litologías. El uso de esta concentración total, empleado como criterio de evaluación, para la determinación de los efectos potenciales de contaminación por metales pesados en suelos, significaría que todas las formas químicas en las que puede encontrarse el metal causan o producen el mismo impacto sobre el medio ambiente, lo que es claramente insostenible. Los metales pesados pueden ser retenidos en los suelos, entre otros, por mecanismos de adsorción: sobre minerales de arcilla, sobre oxihidróxidos metálicos y sobre materia orgánica (1).

Los metales pesados están inmersos dentro de un sistema reactivo gobernado por un conjunto de equilibrios que controlan las concentraciones de dichos metales en la disolución del suelo por medio de reacciones de precipitación-disolución, reacciones ácido base, formación de complejos, reacciones redox, reacciones de cambio iónico y adsorción (2).

Para comprender el comportamiento de los metales pesados en el medio ambiente es necesario obtener información sobre la especiación química de estos elementos, es decir, determinar las formas orgánicas e inorgánicas específicas que forman parte de la concentración total del elemento en solución, al igual que las contenidas en sedimentos. Por medio de la especiación química es posible determinar la biodisponibilidad de los metales para los organismos acuáticos, además de obtener información completa de las condiciones ambientales de los ecosistemas acuáticos y su relación con las diferentes formas en que se encuentran los elementos en la fracción sedimentaria (2).

En el Orinoco medio son escasos los estudios relacionados a metales pesados y en especial a los relacionados con especiación, esto quizás, debido a la dificultad en la logística y difícil acceso a la zona. Es por esta razón que se hizo necesario el desarrollo de esta investigación que permitirá determinar las condiciones ambientales de los sedimentos de este ecosistema, respecto a las concentraciones totales de $\mathrm{Cd}, \mathrm{Cu}, \mathrm{Fe}, \mathrm{Ni}, \mathrm{Mn}, \mathrm{Pb}, \mathrm{Zn}$ y Hg, así como también evaluar su biodisponibilidad utilizando el procedimiento de extracción secuencial.

\section{Materiales y métodos}

El río Orinoco ocupa el tercer lugar entre los ríos más caudalosos del mundo. Su cuenca está compartida geopolíticamente por Venezuela y Colombia y abarca una superficie de $1.080 .000 \mathrm{~km}^{2}$, de los cuales Venezuela posee el $71 \%$ y Colombia el $29 \%$ restante. Por su margen derecha, el Orinoco recibe los aportes de tributarios que drenan el Escudo Guayanés, mientras que por su margen izquierda recibe los ríos que drenan los Andes Colombianos y Venezolanos, al igual que los Llanos ubicados entre los Andes y el Escudo Guayanés. 
El Área de estudio se ubicó específicamente en la zona comprendida entre la desembocadura del río Apure hasta Caicara del Orinoco, Municipio Cedeño del estado Bolívar (figura 1) en un transepto aproximado de 76 $\mathrm{Km}$. Esta zona tiene como característica especial que, en las aéreas aledañas a se está llevando a cabo en la actualidad, la construcción del tercer puente sobre el rio Orinoco, el cual unirá a los estados Guárico y Bolívar a través de las ciudades Cabruta y Caicara del Orinoco. También se encuentran los puertos de embarcaciones de ambas ciudades.

Se recolectaron 28 muestras de sedimentos superficiales en dos muestreos durante los meses de febrero 2001 y abril 2002 utilizando de una draga tipo Diez Laffont de $0,02 \mathrm{~m}^{2}$. Catorce muestras aproximadamente de $1 \mathrm{Kg}$ cada una, fueron recolectadas el 10 de febrero del 2001 y catorce el 16 abril del 2002. Estos meses fueron seleccionados por pertenecer a la temporada de aguas bajas, lo cual facilita la logística de campo y la obtención de las muestras.
Por otra parte, las estaciones de muestreos fueron fijadas con el propósito de cubrir los tres márgenes del rio y las zonas de descargas antropogénicas. Para evitar una posible contaminación y alteración de los resultados, las muestras de sedimentos fueron colocadas en envases secos de polietileno previamente lavados con $\mathrm{HNO}_{3}$ al $10 \%$ y abundante agua desionizada. Las muestras fueron trasportadas refrigeradas hasta los laboratorios del Instituto Limnológico de la Universidad de Oriente.

Los sedimentos fueron mantenidos bajo congelación a $-20^{\circ} \mathrm{C}$, hasta su procesamiento y análisis en el laboratorio de metales traza del Instituto Oceanográfico de Venezuela. Para la determinación de los metales en los sedimentos, una fracción húmeda fue secada a $80^{\circ} \mathrm{C}$ en una estufa PSELECTA (USA), y luego pulverizados en un mortero de porcelana. Las muestras utilizadas para los análisis de mercurio fueron secadas a temperatura ambiente para evitar la pérdida por evaporación del metal.

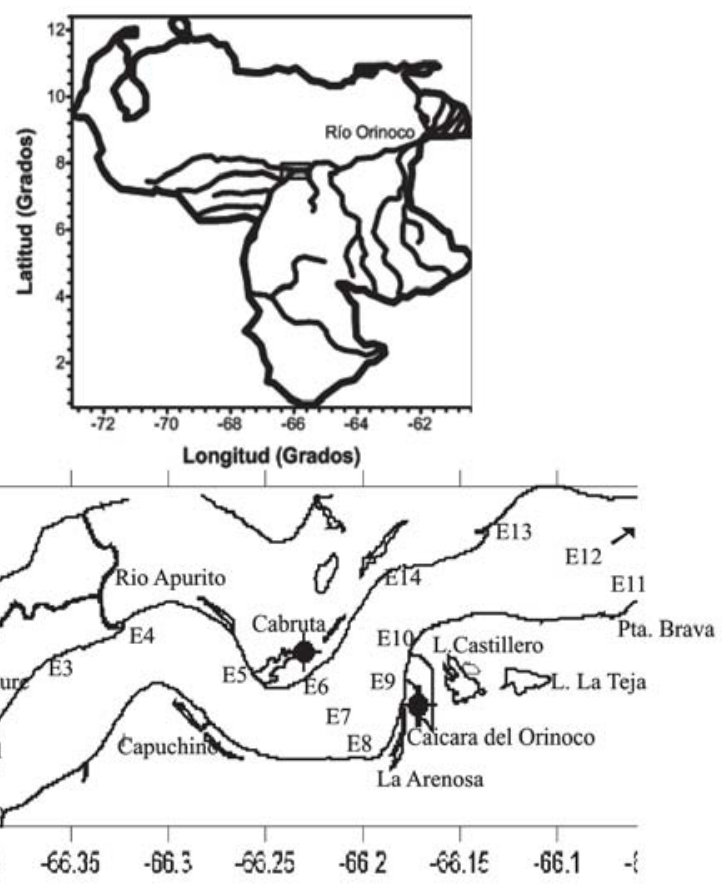

Figura 1. Área de estudio mostrando las estaciones muestreadas en el Orinoco Medio, Venezuela, durante los meses febrero 2001 y abril 2002. 
Para el análisis granulométrico se utilizaron las doce primeras estaciones y se procedió según lo sugerido en (3). Los sedimentos fueron secados a $80^{\circ} \mathrm{C}$ en una estufa $\mathrm{P}$ SELECTA (EUA) y luego pasados través de tamices de 4,00; 2,00; 1,00; 0,85; 0,50; 0,25 y $0,063 \mathrm{~mm}$. Los sedimentos preservados fueron descongelados y secados a $60^{\circ} \mathrm{C}$ en una estufa hasta obtener un peso constante, luego se tamizaron para obtener la fracción menor a $2 \mathrm{~mm}$ y se pulverizaron en un mortero de porcelana, para luego ser almacenados en envases de polietileno previamente lavados hasta los análisis. La materia orgánica total fue calculada por diferencia de peso a través del método de calcinación a $540^{\circ} \mathrm{C}$ descrito en (4). Para ello se utilizó una mufla Marca JELRUS y una balanza analítica Marca Denver Instrument M-10 (USA) con precisión de 0,0001g.

Los carbonatos se determinaron por titulación volumétrica con $\mathrm{NaOH} 0,50$ mol. $^{-1}$ (5). Para ello, se pesó $0,5 \mathrm{~g}$ de sedimentos previamente calcinados, se añadió $15 \mathrm{~mL}$ de

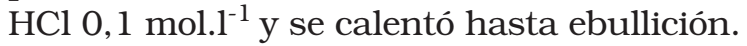
Las muestras se filtraron para eliminar la cantidad de sedimento que obstaculizaba el cambio de color del indicador en el punto final de la titulación y se valoró con $\mathrm{NaOH}$ 0,285 mol. $\mathrm{l}^{-1}$, usando como indicador azul de bromofenol. Los análisis de materia orgánica, y carbonatos se realizaron para determinar las asociaciones estadísticas de los metales con estas variables.

Para los análisis de metales totales, 2 g aproximadamente de muestra fueron pesadas por triplicado en una balanza analítica marca Denver Instrument M-10 (USA) con precisión de 0,0001g, y luego digestadas con una mezcla de $\mathrm{HNO}_{3}$, $\mathrm{HCl}$ y $\mathrm{HClO}_{4}(3: 2: 1)$, según las modificaciones propuestas por (6) utilizando un digestor de Microondas Microdigest 401 de Prolabo (Francia), a $110^{\circ} \mathrm{C}$, por espacio de 30 minutos. Esta fracción (F3) contiene los metales totales en el sedimento. Las cuantificaciones fueron realizadas por espectrofotometría de absorción atómica con llama aire-acetileno y corrector de fondo de deuterio, utilizando un equipo Perkin Elmer modelo 31 10. Para la determinación de mercurio se pesó $1 \mathrm{~g}$ de cada muestra de sedimento seco en un erlenmeyer de $125 \mathrm{~mL}$ y se sometió a digestión con $5 \mathrm{~mL}$ de $\mathrm{HNO}_{3}$ concentrado y luego con $5 \mathrm{~mL} \mathrm{H}_{2} \mathrm{O}_{2}$ al $10 \%$ (v/v). Para la digestión los enlermeyer fueron colocados en un baño de arena por tres horas para regular y mantener la temperatura homogénea en $50^{\circ} \mathrm{C}$. Las soluciones resultantes se filtraron en balones de $25 \mathrm{ml}$ y se enrasaron con agua desionizada. La determinación de mercurio se efectúo por la técnica de vapor frío (CVAAS), mediante la absorción de vapor de mercurio a 253,7 nm, utilizando un equipo de absorción atómica, Perkin Elmer MHS-10, adaptando una celda para el paso de nitrógeno y la inyección de borohidruro de sodio (7).

\section{Especiación de metales}

Se utilizó el método descrito en $(6,8)$. El método descrito consiste en una extracción secuencial de dos pasos: paso uno $(\mathrm{F} 1)=\mathrm{ex}^{-}$ tracción con solución de acido acético 25\% (v/v); paso dos $(\mathrm{F} 3)=$ extracción mezcla azotrópica de $\mathrm{HNO}_{3}: \mathrm{HCl}: \mathrm{HClO}_{4}$ (3:2:1). La primera extracción hecha con ácido acético 25\% fue realizada por 4 horas a temperatura ambiente. Esta fracción (F1) contiene los metales (biodisponibles) adsorbidos en la superficie de las partículas, asociados con los carbonatos y los oxihidróxidos de manganeso reactivos. De manera indirecta, por diferencia entre las fracciones, $\mathrm{F} 3$ (fracción extraída con $\mathrm{HNO}_{3}$ : HCl: $\mathrm{HClO}_{4}$ ) - F1 (ácido acético 25\%), se obtuvo, una segunda fracción (F2) la cual contiene los oxihidróxidos de hierro más resistente y sulfuros metálicos, al igual que los metales asociados a los minerales residuales refractarios y a la materia orgánica.

Para evaluar la calidad analítica de las extracciones de los metales, se utilizó un material estándar de sedimento certificado por la Enviromental Resource Associates: Catálogo número 540, lote 237 (Priority Pollutn/CPL. Soil). La precisión del método de extracción de metales fue significativa, tal 
Tabla 1

Precisión y exactitud del método de extracción de metales pesados totales en el sedimento de referencia, patrón Lot. $N^{\circ}$ 237.Cat. $N^{\circ} 540$

\begin{tabular}{lcccc}
\hline Metal & Valor Exp. $\left(\mu \mathrm{g} \mathrm{g}^{-1}\right)$ Valor Certif. $\left(\mu \mathrm{g} \mathrm{g}^{-1}\right)$ & Rango Acep. $\left(\mu \mathrm{g} \mathrm{g}^{-1}\right)$ & Ds \\
$\mathrm{Fe}$ & 7991 & 7760 & $2890-12600$ & 6,0 \\
$\mathrm{Zn}$ & 87,57 & 87,8 & $67,9-108$ & 0,4 \\
$\mathrm{Mn}$ & 112 & 127 & $101-154$ & 1,0 \\
$\mathrm{Cr}$ & 88,2 & $70,5-106$ & 0,4 \\
$\mathrm{Cu}$ & 108 & $88,7-127$ & 0,6 \\
$\mathrm{Ni}$ & 107 & 55,9 & $43,7-68$ & 0,4 \\
$\mathrm{Cd}$ & 51,7 & 52,4 & $40,3-64,5$ & 0,5 \\
$\mathrm{~Pb}$ & 48,1 & 75,1 & $57,2-93$ & 1,2 \\
$\mathrm{Co}$ & 70,7 & 46,9 & $37,3-56,5$ & 0,2 \\
\hline
\end{tabular}

Lot: Lote; Cat.: Catálogo; Exp.: Experimental; Acep.: Aceptado; Certif.: Certificado. Ds=desviación estándar.

como lo demuestran las desviaciones estándar significativamente bajas para las 5 replicas analizadas y la comparación de los promedios obtenidos con el rango aceptable y el valor del material estándar de sedimento. Los limites de detección para los elementos determinados fueron $0,004 \mu \mathrm{g} \mathrm{g}^{-1}$ para $\mathrm{Cd}$; $0,02 \mu \mathrm{g} \mathrm{g} \mathrm{g}^{-1} \mathrm{Cu} ; 0,01 \mu \mathrm{g} \mathrm{g} \mathrm{g}^{-1} \mathrm{Fe} ; 0,004 \mu \mathrm{g} \mathrm{g} \mathrm{g}^{-1}$ Ni; 0,002 $\mu \mathrm{g} \mathrm{g}^{-1} \mathrm{Mn}$; 0,001 $\mu \mathrm{g} \mathrm{g} \mathrm{g}^{-1} \mathrm{~Pb}$ y 0,002 $\mu \mathrm{g} \mathrm{g}^{-1}$ para $\mathrm{Zn}$. El agua utilizada tanto en la preparación de reactivos, curvas de calibración y blancos de reactivos fue agua desionizada altamente pura (agua calidad NANOPURE de conductividad $18 \mathrm{U} / \mathrm{cm}$ ) obtenida con un equipo NANOPURE UV, Marca Barnstead (USA). El material volumétrico de vidrio utilizado en el laboratorio fue Clase A y los reactivos grado Analítico ultra puro.

Para establecer la existencia o no de contaminación por metales pesados en los sedimentos, se realizaron comparaciones con los niveles propuestos en (9) y otras referencias bibliografías que tienen normas internacionales establecidas. Este tipo de comparación radica en que, uno de los principales problemas al interpretar los resultados e indicar si un sedimento está contaminado en Venezuela, es que se carece de normativas legales que señalen valores consi- derados como contaminantes para los sedimentos (2). Se aplicó análisis de correlación de Pearson a un nivel de significancia $\mathrm{P}<0,05$ para las relaciones entre los metales, materia orgánica y carbonatos y análisis de conglomerados empleando el método de mínima varianza de Ward's y la distancia métrica Euclidiana para determinar asociaciones entre la concentración de los metales y el tamaño de la partícula en el sedimento. Los datos se analizaron con el paquete estadístico STATGRAPHICS Plus 4,1.

\section{Resultados y discusión}

La figura 2, muestra la composición granulométrica de los sedimentos del transepto estudiado en el Orinoco Medio. Las diferentes estaciones (E) presentaron bastante heterogeneidad en cuanto a la textura. El lecho del río Orinoco cerca de la desembocadura del rio Apure está compuesto exclusivamente por partículas muy finas con un alto contenido de lodos (99\%) y un bajo porcentaje de arena media fina (AMF) que alcanza un 1\%. En la E2, la cual no está impactada directamente por la descarga del río Apure, por estar ubicada aguas arriba, los porcentajes son bastantes discrepantes, apreciándose un 98\% de textura de tipo arenosa, distribuidos los valores en 


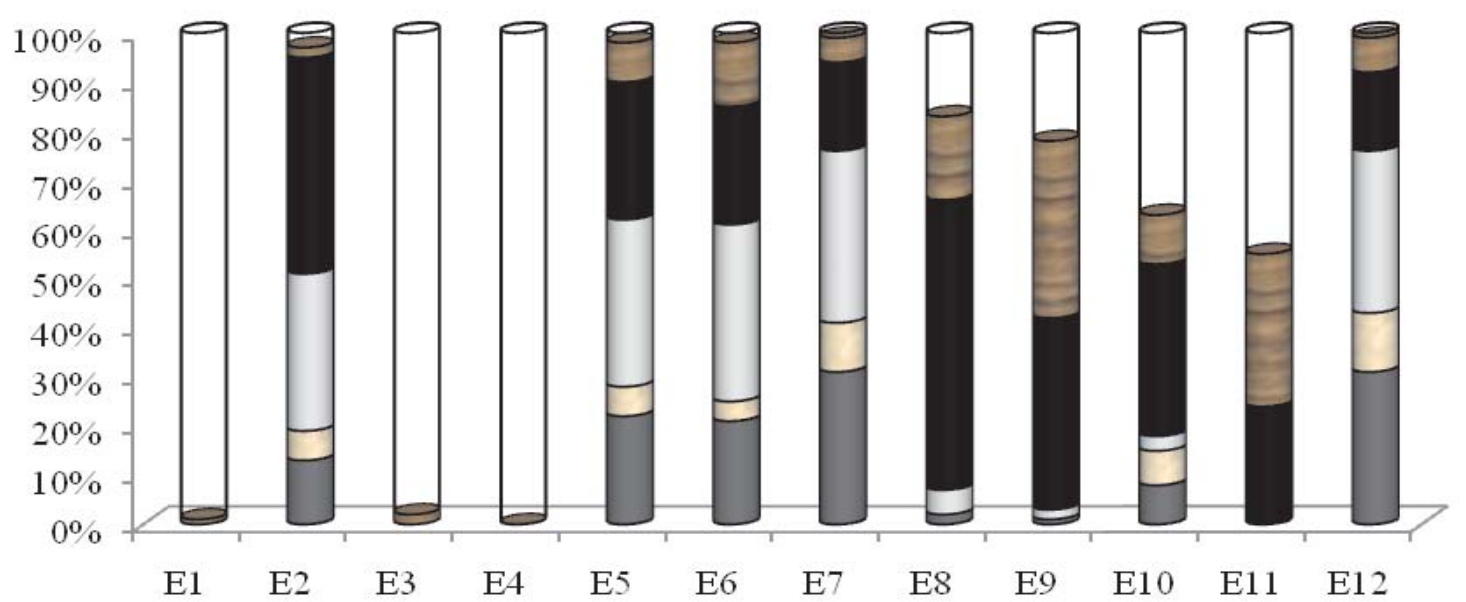

$\square \mathrm{AG} \square \mathrm{AMG} \square \mathrm{AM} \square \mathrm{AF} \square \mathrm{AMF} \square \operatorname{Lodos}$

Figura 2. Características granulométricas (\%) de los sedimentos superficiales del Orinoco Medio, Venezuela ( $\mathrm{AMF}=$ arena muy fina; $\mathrm{AF}=$ arena fina; $\mathrm{AM}=$ arena media; $\mathrm{AMG}=$ arena muy gruesa; $\mathrm{AG}=$ arena gruesa).

un 44\% de AF; $32 \%$ de AM; $13 \%$ de AG y 6\% de AMG y $2 \%$ de AMF, solo un $1 \%$ de lodos fue apreciado. Posterior a la descarga del río Apure sobre las aguas del Orinoco, se observaron variaciones bastantes discrepantes en el resto del área estudiada.

En la E3 y E4 (desembocadura del río Apurito), se observa el incremento de sedimento de grano fino (lodos), causada la acumulación por efecto la formación de una zona de represa de baja energía, originado por el choque de las aguas de estos dos ríos con el cauce principal del Orinoco Medio, que permite una gran sedimentación de las partículas transportadas en las aguas. Posterior a la descarga del río Apure sobre el Orinoco, se aprecia una variación en los porcentajes de cada material encontrándose valores máximos de 31\% AG en la E7; $12 \%$ de AMG en la E12; $36 \%$ de AM en la E6; 59\% de $\mathrm{AF}$ en la E8 y 36\% de AMF en la E9.

Las concentraciones totales $\left(\mu \mathrm{g} \mathrm{g}^{-1}\right) \mathrm{de}$ los metales variaron entre $8871-116759 \mu \mathrm{g}$ $\mathrm{g}^{-1}$ para Fe, $102,45-469,44 \mu \mathrm{g} \mathrm{g}^{-1} \mathrm{Mn} ; 0,93$ - 17,64 $\mu \mathrm{g} \mathrm{g}^{-1} \mathrm{Cu} ; 4,46-17,48 \mu \mathrm{g} \mathrm{g}^{-1} \mathrm{Ni} ; 2,46$

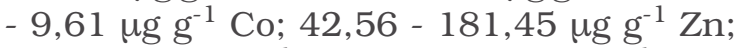
$1,29-8,76 \mu \mathrm{g} \mathrm{g}^{-1} \mathrm{Cr} ; 0,03-0,74 \mu \mathrm{g} \mathrm{g}^{-1} \mathrm{Cd} \mathrm{y}$
0,001 -7,88 $\mu \mathrm{g} \mathrm{g}^{-1} \mathrm{~Pb}$ (tabla 2). Por otra parte, los valores promedios mostraron un gradiente decreciente: Fe (55197) $>\mathrm{Mn}$ $(267,31)>\mathrm{Zn}(85,47>\mathrm{Ni}(9,19)>\mathrm{Cu}(5,60)>$ $\mathrm{Co}(5,27)>\operatorname{Cr}(4,36) \mathrm{Pb}(2,47)>\operatorname{Cd}(0,15)$. No se detecto la presencia de $\mathrm{Hg}$ (tabla 2). Por otra parte los metales totales estuvieron asociados mayoritariamente a las partículas de menor diámetro.

Algunos metales como $\mathrm{Cd}, \mathrm{Cu}, \mathrm{Cr}, \mathrm{Fe}$, $\mathrm{Pb}, \mathrm{Mn}$, Co y Ni están asociados en un mismo grupo a los lodos, arena fina (AF) y a la arenas media fina (AMF), mientras que zinc tiende a estar asociado de igual manera con estos mismos componentes al igual que con la arena media gruesa (figura 3). Estas asociaciones sugieren orígenes similares para estos metales.

La distribución de hierro (figura 4A) a lo largo de la zona estudiada muestra un descenso para el hierro en las concentraciones aguas abajo, mientras que manganeso (figura 4B) presenta 3 picos máximos en las estaciones, E5, E9 y E11 y cobre (figura 4C) en las estaciones, E4 y E11. Níquel (figura 4D) muestra un comportamiento similar a cobre al igual que cobalto (figura $4 \mathrm{E}$ ). Los máxi- 
Tabla 2

Concentraciones totales $\left(\mathrm{gg}^{-1}\right)$ de metales pesados, materia orgánica (\%MOT) y carbonatos en sedimentos superficiales del Orinoco Medio

\begin{tabular}{cccccccccccc}
\hline $\mathrm{E}$ & $\mathrm{Co}$ & $\mathrm{Cu}$ & $\mathrm{Cd}$ & $\mathrm{Cr}$ & $\mathrm{Ni}$ & $\mathrm{Zn}$ & $\mathrm{Mn}$ & $\mathrm{Fe}$ & $\mathrm{Pb}$ & $\mathrm{MOT}$ & Carbonatos \\
\hline 1 & 5,21 & 7,39 & 0,74 & 5,26 & 9,96 & 63,39 & 312,37 & 116759 & 3,43 & 3,23 & 4,06 \\
2 & 3,06 & 1,61 & 0,38 & 1,90 & 4,80 & 42,98 & 102,45 & 58078 & 0,12 & 0,84 & 2,22 \\
3 & 6,43 & 9,51 & 0,16 & 6,85 & $\mathbf{1 2 , 0 9}$ & 108,14 & 415,89 & 96841 & 3,83 & 3,42 & 4,30 \\
4 & 9,61 & $\mathbf{1 7 , 6 4}$ & 0,21 & 8,76 & $\mathbf{1 7 , 4 8}$ & 101,30 & 421,81 & 60508 & 5,88 & 5,18 & 7,08 \\
5 & 4,98 & 5,19 & 0,10 & 4,34 & 9,05 & 83,88 & 244,88 & 42843 & 1,10 & 2,35 & 4,20 \\
6 & 4,16 & 4,04 & 0,05 & 3,65 & 8,50 & 57,55 & 220,05 & 62790 & 0,00 & 1,86 & 3,86 \\
7 & 3,01 & 1,61 & 0,03 & 2,24 & 5,83 & 56,81 & 113,98 & 48275 & $\mathbf{7 , 8 8}$ & 0,85 & 1,67 \\
8 & 4,68 & 4,42 & 0,03 & 5,38 & 8,86 & $\mathbf{1 8 1 , 4 5}$ & 394,01 & 38048 & 2,92 & 2,41 & 4,41 \\
9 & 6,19 & 6,08 & 0,12 & 4,67 & $\mathbf{1 0 , 2 9}$ & 97,12 & 269,30 & 54908 & 0,26 & 3,17 & 4,62 \\
10 & 6,92 & 7,86 & 0,06 & 6,06 & $\mathbf{1 2 , 1 6}$ & 89,39 & 296,76 & 64509 & 3,64 & 3,78 & 6,91 \\
11 & 9,11 & $\mathbf{1 0 , 0 3}$ & 0,09 & 7,61 & $\mathbf{1 4 , 8 4}$ & 135,52 & 469,44 & 33300 & 5,60 & 4,71 & 6,80 \\
12 & 3,82 & 0,93 & 0,04 & 1,62 & 5,15 & 42,56 & 220,89 & 39867 & $\mathrm{LD}$ & 0,69 & 1,21 \\
13 & 4,20 & 1,09 & 0,05 & 1,46 & 5,22 & 70,79 & 109,77 & 47155 & $\mathrm{LD}$ & 0,66 & 1,54 \\
14 & 2,46 & 1,04 & 0,04 & 1,29 & 4,46 & 65,67 & 150,74 & 8871 & $\mathrm{LD}$ & 0,51 & 1,16 \\
Max & 9,61 & 17,64 & 0,74 & 8,76 & 17,48 & 181,45 & 469,44 & 116759 & 7,88 & 5,18 & 7,08 \\
Min & 2,46 & 0,93 & 0,03 & 1,29 & 4,46 & 42,56 & 102,45 & 8871 & 0,00 & 0,51 & 1,16 \\
Prom & 5,27 & 5,60 & 0,15 & 4,36 & 9,19 & 85,47 & 267,31 & 55197 & 2,47 & 2,40 & 3,86 \\
Ds & 2,17 & 4,70 & 0,20 & 2,44 & 3,97 & 38,21 & 123,96 & 26494 & 2,65 & 1,57 & 2,09 \\
$\mathbf{I}$ & $\mathbf{\ldots . . .}$ & $<\mathbf{1 0}$ & $<\mathbf{1}$ & $<\mathbf{2 0}$ & $<\mathbf{1 0}$ & $<\mathbf{1 1 0}$ & & & $<\mathbf{5}$ & & \\
\hline
\end{tabular}

Max= máximo; Min= mínimo; Prom= promedio, Ds= desviación estándar; I= Valor límite para sedimento no contaminado (1). Nota: $\mathrm{Hg}$ no detectado en todo el estudio; LD= límite de detección.

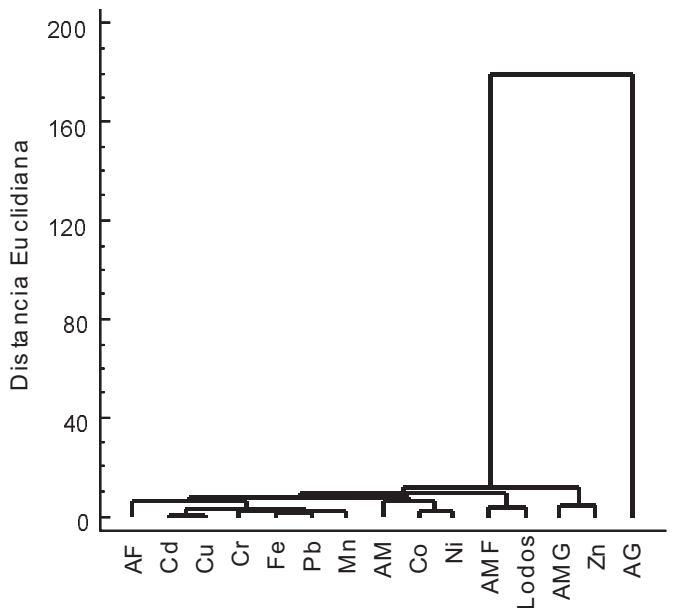

Figura 3. Dendograma de similitud obtenido con el análisis de conglomerado mostrando la asociación entre las concentraciones de metales pesados, composición textural de los sedimentos superficiales del Orinoco Medio, Venezuela. mos de zinc (figura 4F) se observan entre las estaciones, E8 y E11 y cromo (figura 4G) en E4 y E11. Las concentraciones de cadmio (figura $4 \mathrm{H}$ ) siguen una distribución parecida a la del hierro con un máximo en la zona adyacente a la descarga del río Apure, mientras que $\mathrm{Pb}$ (figura 4I) muestra sus máximos en las estaciones E4, E7 y E11. Se observa que la distribución de las concentraciones máximas de todos los metales está afectadas por la zona de descarga de los ríos Apure (E1) y Apurito (E4) y por las zonas aledañas a los puertos de embarcaciones situados en las ciudades de Cabruta (edo Guárico) y Caicara del Orinoco (Edo. Bolívar).

La especiación del hierro para toda la zona (figura 5A) muestra que las mayores concentraciones se encuentran en la fracción F2 (7,50-99,99\%) la cual contiene los oxihidróxidos de hierro más resistente y sul- 


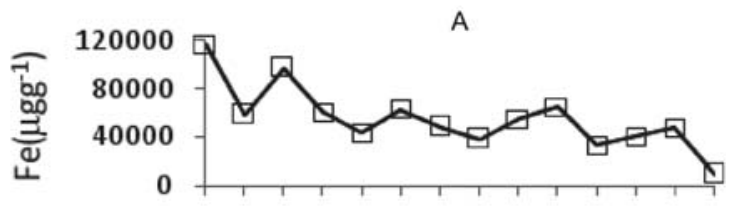

$\begin{array}{llllllllll}1 & 2 & 3 & 4 & 5 & 6 & 7 & 8 & 9 & 1011 \\ 12 & 1314\end{array}$

Estaciones

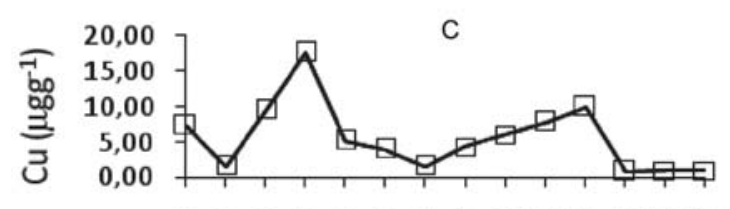

$\begin{array}{lllllllllllllll}1 & 2 & 3 & 4 & 5 & 6 & 7 & 8 & 9 & 10 & 11 & 12 & 13 & 14\end{array}$

Estaciones

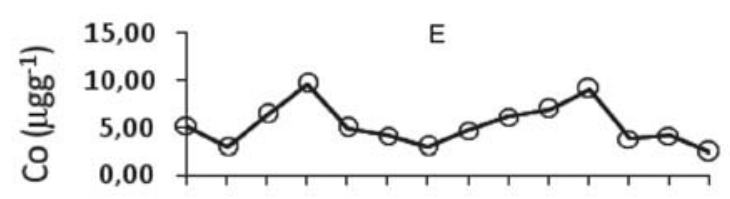

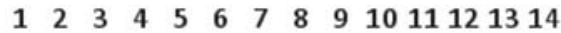

Estaciones

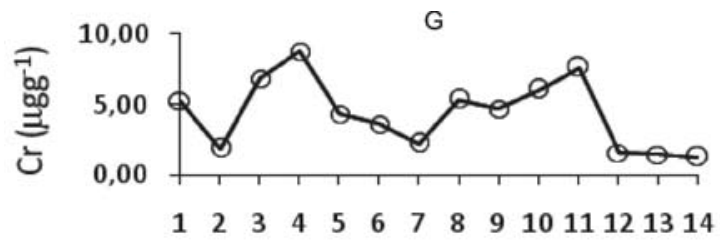

Estaciones

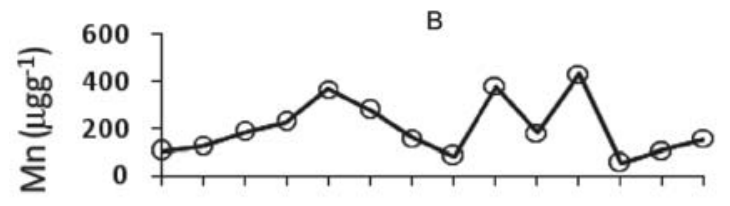

$\begin{array}{lllllllllllllll}1 & 2 & 3 & 4 & 5 & 6 & 7 & 8 & 9 & 10 & 11 & 12 & 13 & 14\end{array}$

Estaciones

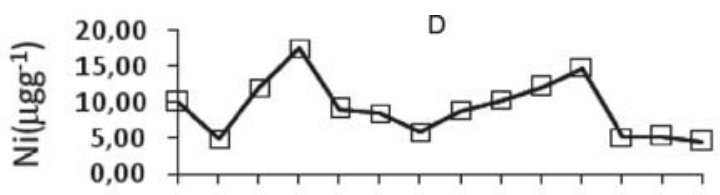

$\begin{array}{llllllllllllll}1 & 2 & 3 & 4 & 5 & 6 & 7 & 8 & 9 & 10 & 11 & 12 & 13 & 14\end{array}$

Estaciones
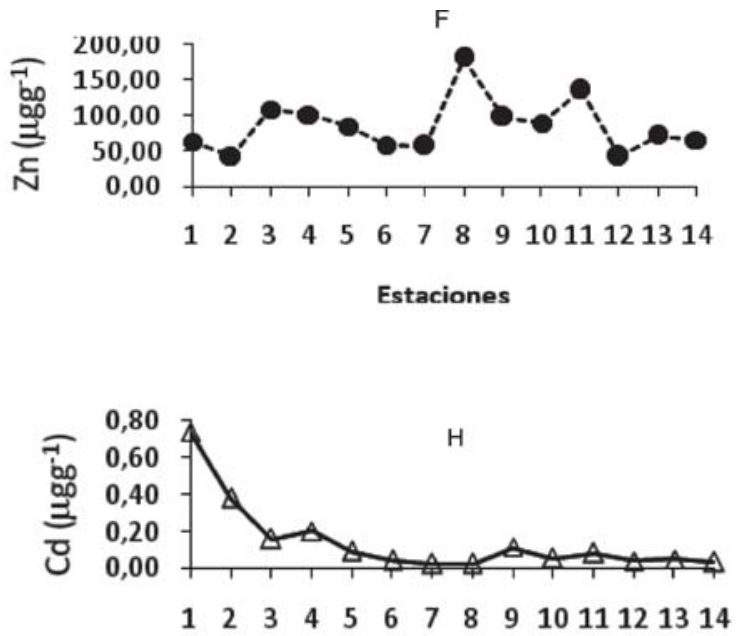

Estaciones

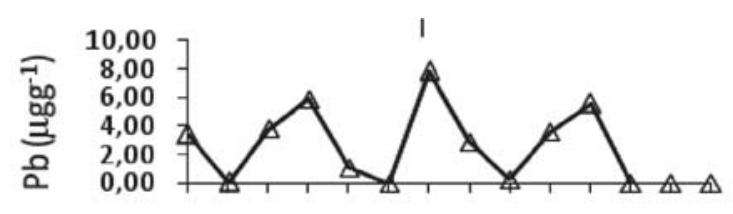

$\begin{array}{llllllllllllll}1 & 2 & 3 & 4 & 5 & 6 & 7 & 8 & 9 & 10 & 11 & 12 & 13 & 14\end{array}$

Estaciones

Figura 4. Distribución de la concentración promedio $\left(\mu \mathrm{g} \mathrm{g}^{-1}\right)$ de metales totales en sedimentos del Orinoco Medio, Venezuela ( $\mathrm{A}=\mathrm{Fe} ; \mathrm{B}=\mathrm{Mn} ; \mathrm{C}=\mathrm{Cu} ; \mathrm{D}=\mathrm{Ni} ; \mathrm{E}=\mathrm{Co} ; \mathrm{F}=\mathrm{Zn} ; \mathrm{G}=\mathrm{Cr} ; \mathrm{H}=\mathrm{Cd} ; \mathrm{I}=\mathrm{Pb})$. 


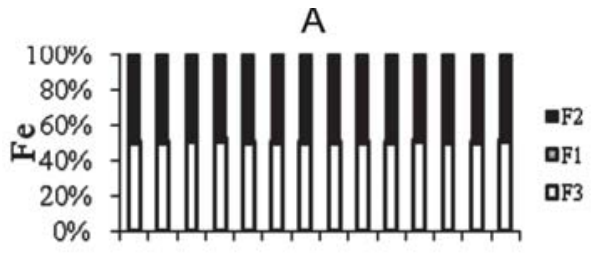

1234567891011121314

Fstaciones

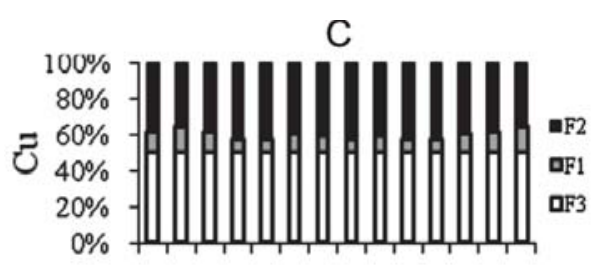

1234567891011121314

Fstaciones

E

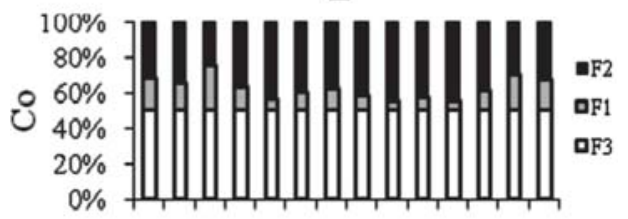

1234567891011121314

Fstaciones

G

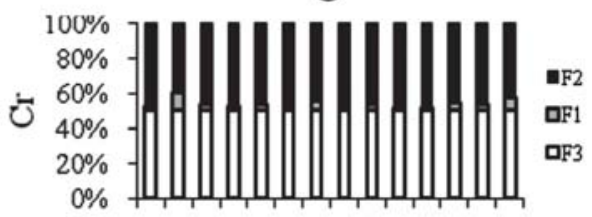

1234567891011121314

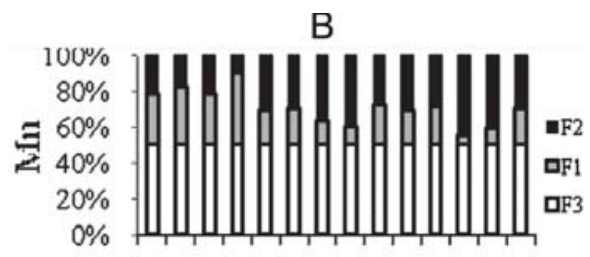

1234567891011121314

Fstaciones

D

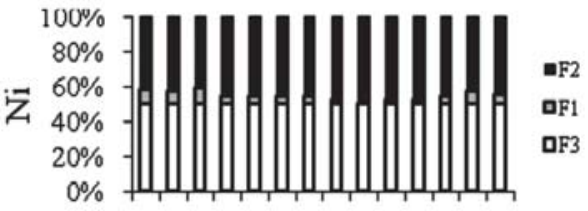

1234567891011121314

Fstaciones

$\mathrm{F}$

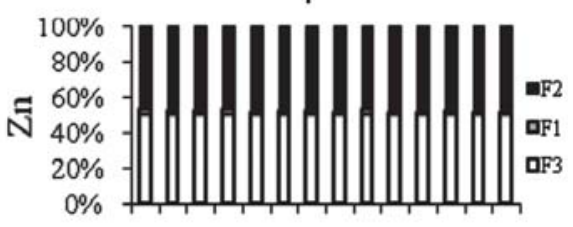

1234567891011121314

Fstaciones

$\mathrm{H}$

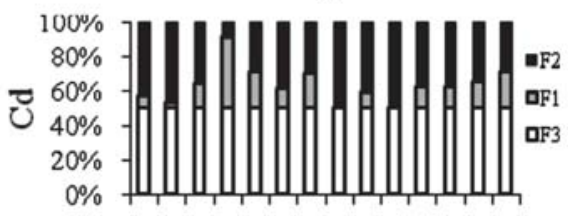

1234567891011121314

Fstaciones

I

Fstaciones

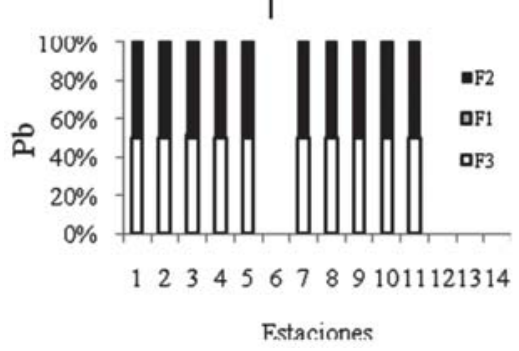

Figura 5. Distribución de de las concentraciones de las diferentes fracciones extraídas en los sedimentos del Orinoco Medio, Venezuela (F1=extracción con ácido acético 25\%(v/v); $\mathrm{F} 3=$ extracción con $\left.\mathrm{HCl}: \mathrm{HNO}_{3}: \mathrm{HClO}_{4}: 3: 1: 1 ; \mathrm{F} 2=\mathrm{F} 3-\mathrm{F} 1\right)$. (A=Fe; $\mathrm{B}=\mathrm{Mn} ; \mathrm{C}=\mathrm{Cu} ; \mathrm{D}=\mathrm{Ni} ; \mathrm{E}=\mathrm{Co}$; $\mathrm{F}=\mathrm{Zn} ; \mathrm{G}=\mathrm{Cr} ; \mathrm{H}=\mathrm{Cd} ; \mathrm{I}=\mathrm{Pb})$. 
Tabla 3

Matriz de correlación de Pearson $(\mathrm{P}<0,05)$, para los parámetros orgánicos e inorgánicos en sedimentos superficiales del Orinoco Medio, Venezuela

\begin{tabular}{lccccccccccc}
\hline & $\mathrm{Co}$ & $\mathrm{Cu}$ & $\mathrm{Cd}$ & $\mathrm{Cr}$ & $\mathrm{Ni}$ & $\mathrm{Zn}$ & $\mathrm{Mn}$ & $\mathrm{Fe}$ & $\mathrm{Pb}$ & $\mathrm{MOT}$ & Carbonatos \\
\hline $\mathrm{Co}$ & 1,00 & & & & & & & & & & \\
$\mathrm{Cu}$ & 0,92 & 1,00 & & & & & & & & & \\
$\mathrm{Cd}$ & $-0,03$ & 0,14 & 1,00 & & & & & & & & \\
$\mathrm{Cr}$ & 0,93 & 0,94 & 0,05 & 1,00 & & & & & & & \\
$\mathrm{Ni}$ & 0,97 & 0,97 & 0,01 & 0,98 & 1,00 & & & & & & \\
$\mathrm{Zn}$ & 0,51 & 0,41 & $-0,29$ & 0,65 & 0,53 & 1,00 & & & & & \\
$\mathrm{Mn}$ & 0,83 & 0,77 & $-0,02$ & 0,91 & 0,84 & 0,77 & 1,00 & & & & \\
$\mathrm{Fe}$ & 0,03 & 0,25 & 0,75 & 0,21 & 0,15 & $-0,24$ & 0,11 & 1,00 & & & \\
$\mathrm{~Pb}$ & 0,41 & 0,46 & $-0,04$ & 0,48 & 0,47 & 0,29 & 0,35 & 0,05 & 1,00 & & \\
$\mathrm{MOT}$ & 0,96 & 0,93 & 0,11 & 0,97 & 0,98 & 0,55 & 0,85 & 0,21 & 0,42 & 1,00 & \\
Carbonatos & 0,91 & 0,84 & $-0,04$ & 0,91 & 0,93 & 0,58 & 0,76 & 0,05 & 0,34 & 0,94 & 1,00 \\
\hline
\end{tabular}

$>0,50=$ significativo; $r>0,70$ altamente significativos.

furos metálicos, al igual que los metales asociados a los minerales residuales refractarios y a la materia orgánica. Con esta ultima la correlación fue de $\mathrm{r}=0,21$ (tabla 3), por lo que el Fe total no está asociado a materia orgánica, tampoco a carbonatos $(\mathrm{r}=0,05)$ y podría encontrarse como oxihidróxidos resistentes, y/o materiales refractarios. En todas las estaciones la cantidad de Fe obtenido en la suma de las fracciones F2+F3 supera en un $98 \%$ del total de las concentraciones determinadas, lo que corrobora el origen litogénico del Fe total, debido a las características mineralógicas presentes en la zona. Las concentraciones determinadas indican que solo en un rango 0,04-1,97\% de Fe está presente en la fracción biodisponible. Esto es confirmado por la baja correlación entre Fe y los carbonatos $(r=0,33)$.

Izquierdo y col. (1997), reportan concentraciones similares en los pantanos salados en España, y explican que debido a que este es uno de los elementos más comunes en la corteza terrestre es frecuente encontrar valores elevados en la fracción residual (10). En sedimentos del río Jacuí, Brasil, se reportan valores de $1,38 \%$ de Fe total en la fracción biodisponible (11). Para los com- puestos de hierro total, las formas reducibles son predominantes y la mayor porción se observa en la fracción residual (12).

El comportamiento del manganeso total (figura 5B), que en la naturaleza generalmente está asociado a hierro, muestra distribución en las fracciones diferente a este elemento, lo cual es corroborado por la baja correlación entre estos dos elementos $(r=0,11)$. La mayor cantidad asociada a la fracción F2 (7,75 - 66,34\%) con una correlación estadística significativa a la materia orgánica $(r=0,85)$, sin embargo, valores entre 4,15$71,59 \%$ están biodisponible (F1), adsorbidos a la superficie de las partículas y asociados con los carbonatos, formando también parte de los oxihidróxidos de manganeso reactivos.

Con los carbonatos la correlación fue de $r=0,76$, confirmando la asociación del manganeso a estos compuestos. Aproximadamente un porcentaje superior a $18 \%$ se encuentra asociado a sulfuros (F2). En (11) se reporta cerca de un 43,49\% en la fracción asociada a los carbonatos y casi un $27,58 \%$ asociado a la fracción residual.

La especiación del cobre total es similar a manganeso total (figura 5C), sin embargo, 
los porcentajes en la fracción biodisponible oscilan entre 1,05- 14,97\% del cobre total. A pesar de que el cobre total esta significativamente correlacionado con los carbonatos $(\mathrm{r}=0,84)$ los porcentajes son menores que en F2 (4,20-85,03\%). La correlación entre el Cu total con la materia orgánica $(r=0,93)$ sugiere una mayor proporción asociada a la materia orgánica, sulfuros y a minerales residuales. En (13) se reporta para sedimentos del sistema de ríos Tietê-Pinheiros en Brasil, que el $\mathrm{Cu}$ total estuvo fuertemente asociado a los sulfuros y la materia orgánica, situación similar a lo reportado en esta investigación. Según lo señalado en (12) se determinaron cantidades muy pequeñas de $\mathrm{Cu}$ en la fracción biodisponible la cual representa casi un $3,45 \%$ del $\mathrm{Cu}$ total valores inferiores a los de la presente investigación.

La especiación de níquel total (Figura 4D) mostró sus máximos porcentajes en los oxihidróxidos de hierro más resistente y sulfuros metálicos, fracciones F2 $(22,85-$ $91,36 \%)$ y F3, con valores menores a entre 0-12,10\% en la fracción biodisponible la cual representa, metales que están adsorbidos en la superficie de las partículas, asociados con los carbonatos y los oxihidróxidos de manganeso reactivos $(r=0,84$ entre Ni-Mn). También a la fracción (F2) que contiene los oxihidróxidos de hierro más resistente y sulfuros metálicos. Sin embargo la mayor correlación del níquel con la materia orgánica $(r=0,98)$ en comparación con Fe $(\mathrm{r}=0,15)$ sugiere que, el níquel se encuentra mayormente asociado a la materia orgánica y no a los minerales refractarios.

Las concentraciones de cobalto total (figura 5E) mostraron sus máximos en la fracción residual (F2) con rangos que van desde 16,76-85,48\%. Sin embargo, debido el alto coeficiente de correlación observada entre cobalto y la materia orgánica $(r=0,96)$ pareciera estar correlacionado en casi su totalidad a materia orgánica y no a sulfuros ni a compuestos de hierro reactivos, debido a la baja correlación entre Co y Fe $(r=0,03)$, tabla 3. Un valor entre un rango de 6,40-
$33,06 \%$ fue determinado en fracción biodisponible. La correlación altamente significativa mostrada entre Co-carbonatos $(\mathrm{r}=0,91)$; Co-Mn $(\mathrm{r}=0,83)$ confirma la asociación a la fracción de oxihidróxidos de Mn reactivos y a carbonatos. El comportamiento observado por el cobalto total fue diferente al reportado por (14) para el lago de Tuskegee en Alabama (USA), quienes indicaron que las máximas concentraciones de Co total fueron encontradas en la fracción asociada a carbonatos (F1). También diferente a lo señalado en (15) para sedimentos del rio Tigris (Reino de Tonga), quienes indican que el cobalto no fue detectado en la fracción disponible. Para sedimentos del río de Jacuí, Brasil se señala que las mayores concentraciones se encuentran en la fracción residual, con pequeñas cantidades en las fracciones biodisponibles, siendo casi un $20 \%$ del Co total (11).

Como se puede observar hay comportamientos diferentes en los ecosistemas. Esto pudiera ser debido la composición mineral del sedimento, al respecto (16) indica que, el cobalto es un elemento que es raro encontrarlo aislado en la naturaleza. Se encuentra en la corteza en un $2,5 \times 10^{-3 \%}$ en peso, donde frecuentemente asociado con minerales de níquel (forbesita $\left[(\mathrm{Ni}, \mathrm{Co}) \mathrm{HAsO}_{4}\right]$, siegenita $\left[\left(\mathrm{Co}, \mathrm{Ni}_{3} \mathrm{~S}_{4}\right]\right)$, plata (yacimientos hidrotermales:hauchecornita [(Ni, Co, $\left.\mathrm{Bi})_{4}(\mathrm{~S}, \mathrm{Sb})_{3}\right]$ ), plomo (galena $\mathrm{PbS}$ ), cobre (carrollita $\mathrm{CuCo}_{2} \mathrm{~S}_{4}$, junto a calcosina $\left(\mathrm{Cu}_{2} \mathrm{~S}\right)$ y calcopirita $\left(\mathrm{FeCuS}_{2}\right)$, bloquita [(Ni, $\mathrm{Co}, \mathrm{Cu}$ ) $\mathrm{Se}_{2}$ ), hierro (vivianita $\left[\mathrm{Fe}_{3}(\mathrm{PO} 4)_{2} \cdot 8 \mathrm{H}_{2} \mathrm{O}\right.$, vallamaninita [(Ni, Cu, Co, $\left.\mathrm{Fe})(\mathrm{S}, \mathrm{Se})_{2}\right]$, mispiquel [FeAsS]).

La distribución del zinc total (figura 5F) muestra sus máximos valores en la fracción F2, representando entre 22,75-98,89\% del $\mathrm{Zn}$ total determinado. Un porcentaje entre 0,86-3,84\% se observa en la fracción F1. Una fracción de $\mathrm{Zn}$ total asociada a la materia orgánica es sugerida debido a la correlación significativa entre ambos $(r=0,58)$ igualmente a oxihidróxidos de $\mathrm{Mn}(\mathrm{r}=0,77$, tabla 3). En sedimentos de río del Valle de 
Mezica (Slovenia) se señala que aproximadamente un $90 \%$ del $\mathrm{Zn}$ total se encuentra en la fracción residual y menos del 10\% en la fracción biodisponible (16). En (11) también señalan para río Jacuí, Brasil, un 74,94\% para la fracción residual y un $10,55 \%$ en la fracción biodisponible. En sedimentos del Lago Tuskegree en USA, el Zn presenta una mayor tendencia a estar asociado a las especies oxidables, al igual que a las formas reducibles (óxidos de Fe y Mn) y una pequeña cantidad asociada a los carbonatos (14), resultado que está acorde con lo observado en esta investigación. En sedimentos del río Anyang (Corea del Sur) Zn se reportó que $60 \%$ del $\mathrm{Zn}$ total se encontraba en la fracción intercambiable, indicando que altas concentraciones en esta fracción es un indicativo de descarga de aguas de desechos cercanas a la zona en estudio (12).

La tendencia del cromo total (figura 4G) fue a estar asociado a las fracciones F2 y F3 representando más del 95\% del cromo total. Los valores en F2 fueron de 2,56-95,49\%.Debido a las correlaciones observadas entre este metal con el hierro $(r=0,21)$ y la materia orgánica $(r=0,97)$, se sugiere que el Cr total está presente en mayor proporción en la fracción de la materia orgánica que en los oxihidróxidos de hierro reactivo. No se descarta una proporción asociada a los sulfuros metálicos minerales residuales.

Respecto a la fracción biodisponible esta muestra una distribución bastante irregular $(\mathrm{F} 1)$, porcentajes entre 1,03-5,08\% son observados cerca de la zona de descarga del río Apure y en algunas estaciones aguas abajo. La buena correlación entre el Cr total con los carbonatos $(\mathrm{r}=0,91)$ y con $\mathrm{Mn}$ $(r=0,91)$ sugiere que el cromo está en está fracción como carbonatos y como oxihidróxidos de Mn reactivos. En (13) se señala que, en los sedimentos del sistema de ríos TietêPinheiros, Brasil, el Cr estuvo presente en la fracción residual, sin embargo, en zonas afectadas antropogénicamente, estuvo mayormente asociada a la materia orgánica. Igualmente en (11) se señala que, el Cr total se encuentra en un $80 \%$ en la fracción residual y un rango entre $15-25 \%$ asociado a la materia orgánica.

El comportamiento del Cd total (figura $4 \mathrm{H}$ ) es diferente a los otros metales estudiados, quizás debido a que, su origen en la naturaleza es mayormente antropogénico, ya que es utilizado en la fabricación de los fertilizantes. Del Cd total, la fracción F 1 tuvo valores entre $0-22,97 \%$ y el resto tuvo asociado a las fracciones F2 y F3, lo cual representa entre 2,03-85,44\%. La correlación altamente significativa con el $\mathrm{Fe}$ (tabla 3) sugiere que el Cd podía estar asociado a oxihidróxidos de Fe. Esta observación es corroborada por la baja correlación entre el Cd total con $\mathrm{CaCO}_{3}(\mathrm{r}=-0,04)$ y con $\mathrm{Mn}(\mathrm{r}=-0,02)$. En el rio Orinoco la presencia del cadmio puede estar relacionado a aguas de descargas, así como, al uso de fertilizantes en la agricultura que se desarrolla en las zonas aledañas de su cauce. En sedimentos de pantanos en Latvia (Republica de Latvijas) se ha reportado una gran afinidad del Cd por la fracción de carbonatos, señalando que este elemento tiene un potencial impacto en los procesos biológicos y en los ecosistemas acuáticos (17). Por otra parte en sedimentos del mismo río evaluado, se encontraron valores de $23 \%$ en la fracción biodisponible y un $22 \%$ asociado a los óxidos de $\mathrm{Fe} / \mathrm{Mn}$, así como, un $27 \%$ a la fracción residual (18). En (12 y 13) se señala que, el Cd está asociado en su mayoría a la fracción biodisponible en el río Tietê-Pinheiros, Brasil y en sedimentos del río Anyang (Corea del Sur). Las concentraciones de Cd presente en cualquier ecosistema pueden tender a bioacumularse y biomagnificarse por lo que siempre es necesario vigilar y estudiar a detalle su comportamiento, para así verificar y evitar una posible contaminación.

El plomo total (figura 5I) está presente en casi su totalidad en la fracciones F2 y F3. En la fracción F2 los valores oscilaron de $7,50-99,29 \%$, mientras que en la fracción $\mathrm{F} 1$ los porcentajes fueron bastante bajos infe- 
riores a $1,78 \%$, a pesar que la presencia de este metal en los ecosistemas es de origen antropogénico, y que se adsorben débilmente a la superficie de partícula tal como lo señala en (9). Las especies químicas de $\mathrm{Pb}$ son fuertemente adsorbidas por óxidos de Fe y Mn según (20).

$\mathrm{El} \mathrm{Pb}$ a diferencia de los demás metales estudiados, no muestra ninguna correlación con los otros metales, ni con materia orgánica ni carbonatos, sugiriendo un origen diferente probablemente en minerales refractarios o en otras fuentes. En sedimentos de río de Latvia determinaron $16 \%$ de $\mathrm{Pb}$ en la fracción biodisponible y $44 \%$ en la fracción residual (18). En el río Jacuí, Brasil, reportaron concentraciones bastante bajas en la fracción biodisponible lo cual represento cerca del 1\% (11). La mayor concentración estuvo en la fracción residual, resultados consistentes con lo observado en esta investigación. Debido a que no se detectó correlación alguna con $\mathrm{Fe}, \mathrm{Mn}$, Ni con materia orgánica se podría sugerir que el $\mathrm{Pb}$ presente en los sedimentos del Orinoco Medio podría estar formando parte de minerales refractarios, lo cual permite inferir que para su libración se necesitarían condiciones muy drásticas.

De acuerdo a lo arrojado por el diagnostico realizado y tomando en consideración la comparación de los valores de concentraciones totales obtenidos y los niveles existentes en la literatura para sedimentos no contaminados, se concluye que, hay intervención antropogénico en algunas estaciones de la zona estudiada, especial para metales como $\mathrm{Cu}, \mathrm{Ni}$, $\mathrm{Zn}$ y $\mathrm{Pb}$ quienes muestran valores que se encuentran por encima a los reportados para sedimentos no contaminados (tabla 2).

\section{Conclusiones}

Las concentraciones promedio de los metales pesados mostraron un gradiente decreciente $\mathrm{Fe}(55197)>\mathrm{Mn}(267,31)>\mathrm{Zn}$ $(85,47>\mathrm{Ni}(9,35)>\mathrm{Cu}(5,77)>\mathrm{Co}(5,27) \mathrm{Cr}$ $(4,36) \mathrm{Pb}(2,65)>\mathrm{Cd}(0,15)$. No se detecto la presencia de Hg. Por otra parte están asociados a las partículas de menor diámetro como, lodos y arenas finas. La mayoría de los metales presentaron sus máximas concentraciones en las fracciones en las cuales los metales están asociados a materia orgánica, oxihidróxidos de hierro reactivo, sulfuros metálicos y materia orgánica $(\mathrm{F} 2+\mathrm{F} 3)$.

En la fracción F2 los rangos variaron entre $(7,50-99,29 \% \mathrm{Fe}, 7,75-66,34 \% \mathrm{Mn}$, $22,55-98,89 \%$ Zn, 22,85-91,36\% Ni, 4,20$85,03 \% \mathrm{Cu}, 16,76-85,48 \%$ Co, $12,56-95,49$ Cr, 7,50-99,29\% Pb, 2,03-85,48\% Cd), sin embargo, los altos coeficientes de correlación mostrados por los metales con la materia orgánica, en comparación con hierro (excepto $\mathrm{Cd}$ y $\mathrm{Pb}$ ), sugiere que, los metales se encuentran en una mayor asociación con la materia orgánica.

Todos los metales estuvieron presentes en la fracción biodisponible aunque en bajos porcentajes. Los rangos variaron entre (0,04-1,97\% Fe; 4,15-71,59\% $\mathrm{Mn}$; 0,86-3,83\% Zn; 0-12,10\% Ni; $1,05-14,97 \%$ $\mathrm{Cu} ; 6,40-33,06 \% \mathrm{Co} ; 1,03-5,08 \% \mathrm{Cr}$; 0-1,78\% Pb; 0-22,97\% Cd). Cu, Ni, Zn y Pb, presentan valores superiores a los reportados en la literatura para sedimentos no contaminados en algunas estaciones, lo que hace suponer que, estas zonas están impactadas antropogénicamente.

Por otra parte aunque los metales están asociados en mayor proporción a las fracciones F2 y F3, todos están presentes en la fracción biodisponibles para los organismos. Se ha planteado que las formas que interesan desde el punto de vista ambiental son las móviles y las biodisponibles. En este caso, algunas formas naturales, por ejemplo, sulfuros y carbonatos, tienden a movilizarse por ligeros cambios de las condiciones ambientales.

De acuerdo a este diagnostico, se podría concluir que si los impactos antropogénicos llegaran a incrementarse, niveles mayores podrían estar biodisponibles para los organismos acuáticos que viven en este eco- 
sistema. Todo esto podría traer a futuro problemas de bioacumulación y biomagnificación. Los metales pesados estudiados en los sedimentos superficiales del Orinoco Medio, indican una biodisponibilidad en el orden siguiente: $\mathrm{Fe}, \mathrm{Zn}$ y $\mathrm{Pb}<5 \%$; $\mathrm{Cu}$, Ni y $\mathrm{Cr}<20 \%$; Co y $\mathrm{Cd}<50 \%$ y $\mathrm{Mn}<72 \%$.

\section{Agradecimientos}

Los autores agradecen a FUNDACITE GUAYANA toda la colaboración prestada para el desarrollo de esta investigación a través del financiamiento del proyecto 000606.

\section{Referencias bibliográficas}

1. VILAR S., GUTIÉRREZ A., ANTEZANA J., CARRAL P., ÁLVAREZ A. Bol Geol Min 114 (4): 453-461. 2003.

2. ANDREAE M. The important of chemical speciation. (Eds). Berhand $\mathrm{M}$, Brickman $\mathrm{F}$. y Sadler DK. Springer-Vergag, Berlin (Germany). 301-335. 1986.

3. MÁRQUEZ A., MARTÍNEZ G., SENIOR W., GONZÁLEZ A. Bol Inst Oceanogr Venezuela 49 (1): 3-16. 2010.

4. GONZÁLEZ H., M. RAMIREZ. J Geochem Explore 52 (1-2): 183-192.

5. VOGEL. 1960. Quimica Analitica Cuantitativa. Vol 1. (Eds). Kapelusz, S.A Buenos Aires (Argentina). 811 p. 1995.

6. LEWIS B., LANDING W. Mar Chem 40: 105-114. 1992.
7. EASTERLING D., HOIVANITZ E., STREET K. Analytical Letters 33 (8):1665-1689. 2000.

8. LANDING W., LEWIS B. Marine Particles: Analysis and Characterization. (Eds. Hurd D. Spencer D. (Eds.) American Geographysical Union. Washington D.C. (USA). pp. 263-272. 1991.

9. SADID, M.. Toxic Metal Chemistry in Marine Environments. Pergamon .Mercel. Dekker, Inc. New Cork (USA). 390 p. 1992.

10. IZQUIERDO C., USERO J., GRACIA. I. Mar Pollu Bull 34 (2): 123-128. 1997.

11. TEIXEINA E., ORTIZ L., ALVES M., SÁNCHEZ. J Envir Geol 41: 145-154. 2001.

12. LEE S.; WON MOON J., SOO MOON H. Envir Geochem and Health 25: 433-452. 2003.

13. DA SILVA I., ABATE G., LICHTIG J., MASINI J Applied Geochem 17: 105-116. 2002.

14. IKEM A., EGIEBOR O., NYAVOR K. Water Air and Soil Pollu 149: 51-75. 2003.

15. BAHATTIN G., GÜLSEN Ö. Chem Speciat and Bioav13 (1):27. 2001.

16. SVETE P., MilACIC R., PIHLAR Y. J Environ Monit 3: 586-590. 2001.

17. KLAVINS M., VIRCAVS M. Boreal Envir Res 6: 297-306. 2001.

18. FERGUSSON, J. The heavy metals elements chemistry environmental impact and health effects. Pergamon Press, Oxford (England). 175-18 pp. 1990. 